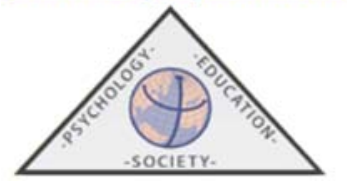

\author{
ISSN $2171-2085$ (print)/ISSN $1989-709 x($ online)
}

\title{
Búsqueda de sensaciones e impulsividad como predictores de la agresión en adolescentes
}

\author{
José J. GÁZQUEZ ${ }^{1}$, Ma Carmen PÉREZ-FUENTES, Ma Mar MOLERO y \\ Ma Mar SIMÓN \\ Universidad de Almería, España \\ (Recibido 15 Abril, 2016; Aceptado 16 Junio, 2016)
}

RESUMEN: La adolescencia es una etapa, en la que la impulsividad y la búsqueda de sensaciones se presentan como dos factores que confluyen con un amplio abanico de cambios, inherentes al desarrollo adolescente. La impulsividad y la búsqueda de sensaciones han sido frecuentemente vinculadas a comportamientos de riesgo en adolescentes, y especialmente afines a la agresividad. Con el presente trabajo se pretende analizar el valor predictivo de variables relacionadas con la búsqueda de sensaciones y la impulsividad, en relación a la adopción de diferentes formas de agresión (agresión física, agresión verbal, ira y hostilidad), en adolescentes. Para ello, se seleccionó una muestra de 822 alumnos de secundaria, y se aplicaron la Escala de Búsqueda de Sensaciones, la Escala de Impulsividad Estado (EIE) y el Cuestionario de Agresión (AQ). Los resultados obtenidos, evidencian la existencia de correlaciones positivas entre los factores de búsqueda de sensaciones e impulsividad, con respecto a las modalidades de agresión analizadas. Por otro lado, los análisis de regresión múltiple evidencian el valor predictivo de los factores de búsqueda de sensaciones e impulsividad, para las diferentes manifestaciones de la conducta agresiva. Más concretamente, destaca la presencia de los componentes de la impulsividad en los modelos explicativos de la agresión física y verbal, y el factor Atencional como el mejor predictor de la ira y la hostilidad.

Palabras clave: Impulsividad, búsqueda de sensaciones, agresión, predictores, adolescentes.

Sensation seeking and impulsivity as predictors of aggression in adolescents

ABSTRACT: Adolescence is a stage in which impulsivity and sensation seeking are presented as two factors that come together with a wide range of changes inherent to adolescent development. Impulsivity and sensation seeking have often been linked to risky behavior in adolescents, especially related to aggressiveness. With this paper is to analyze the predictive value of variables related to sensation seeking and impulsiveness, in relation to the adoption of different forms of aggression (physical aggression, verbal aggression, anger and hostility) in adolescents. To do this, a sample of 822 high school students were selected, and the Sensation Seeking Scale, the State Impulsivity Scale (EIE) and Aggression Questionnaire (AQ), were applied. The results obtained show the existence of positive correlations between factors of sensation seeking and impulsivity, regarding the modalities of aggression analyzed. On the other hand, multiple regression analysis shows the predictive value of factors sensation seeking and impulsiveness, for the different manifestations of aggressive behavior. More specifically, the presence of components of impulsivity in the explanatory models of physical and verbal aggression, and attentional factor, as the best predictor of anger and hostility.

Keywords: Impulsiveness; sensation seeking; aggression; predictors; adolescents.

${ }^{1}$ Correspondencia: José Jesús Gázquez Linares. Dtpo de Psicología. Universidad de Almería. jlinares@ual.es. 
La etapa de la adolescencia se caracteriza por una prematura experimentación de nuevas experiencias y sensaciones (Azpiazua, Esnaola, y Sarasa, 2015; Galvan, 2010). Zuckerman, Persky, Link, y Basu (1994), definen la búsqueda de sensaciones como el deseo de experimentar variadas y complejas experiencias, y la tendencia a implicarse en riesgos físicos y sociales, por el simple deseo de disfrutar de ello. Muñoz, Navas, y Graña (2005), sugieren que los principales factores de riesgo psicológicos para explicar la conducta antisocial son variables vinculadas a la búsqueda de sensaciones, tales como desinhibición y búsqueda de excitación, y la impulsividad. Moral y Ortiz (2011), encontraron correlaciones entre la conducta antisocial en adolescentes y la búsqueda de sensaciones, en sus cuatro dimensiones: desinhibición, búsqueda de excitación, búsqueda de emociones y susceptibilidad al aburrimiento.

La conducta agresiva, tal y como la define Buss (1961), consiste en una respuesta que aporta estímulos dañinos a otro organismo. A partir de esta primera aproximación, se deduce el efecto nocivo de la agresión, aunque desde una perspectiva contextualizadora, se reconoce que determinados actos agresivos pueden ser reprobados en determinadas situaciones y ser aceptables en otras (Bandura, 1976). En esta línea, la agresión se traduce en toda aquella actividad a través de la cual una persona busca infringir daño a otra persona que está motivada a evitarlo. Por tanto, la dimensión subjetiva de la agresión, en la que se configura como una conjunción de ideas, emociones y conductas, ha ido evolucionando hasta concretarse términos como la agresividad, la ira o la hostilidad (Calvet et al., 2012). De este modo, queda patente en el uso posterior de conceptualizaciones en el estudio de la agresividad, donde se diferencia entre agresión física, agresión verbal, ira y hostilidad (Inglés et al., 2014; McKay, Perry, y Harvey, 2016). En hallazgos recientes (Rubio-Garay, Carrasco, y Amor, 2016), se sugiere que la ira y la hostilidad contribuyen positivamente a la agresión física y verbal. En el estudio de Chen, Coccaro, y Jacobson (2012), la hostilidad se asoció positivamente con la agresión física, relacional y verbal, y además se detectó una interacción significativa entre la impulsividad y la hostilidad para la agresión en general. En ocasiones la hostilidad puede llegar a presentar diferentes manifestaciones como aquellos casos en los que se traduce en sentimientos de rechazo hacia la escuela (Inglés, Gonzálvez-Maciá, García-Fernández, Vicent, y MartínezMonteagudo, 2015) con efectos sobre el rendimiento académico (Álvarez et al., 2015).

Otra de las variables estudiadas en torno a la conducta agresiva es la impulsividad (Archer y Webb, 2006). Ésta se define como una expresión del comportamiento desinhibido, caracterizado por la faltade control de la conducta (Cardoso-Moreno, Tomás-Aragonés, y Rodríguez-Ledo, 2015). De ahí, que autores como Neumann, Barker, Koot, y Maughan (2010) analizaran el efecto de la impulsividad en el desarrollo de la conducta antisocial en adolescentes, mostrando con sus resultados que la impulsividad, era una de las variables que más se asociaba con conductas de riesgo, en ambos sexos. También en sujetos con trastornos psiquiátricos, se ha podido comprobar que existe una estrecha relación entre la agresión, física y verbal, y altos niveles de impulsividad (Coccaro, Lee, y McCloskey, 2014). De hecho, existen autores que obtienen resultados que apoyan la inclusión de la agresión verbal como criterio diagnóstico de trastornos psiquiátricos, como el Trastorno Explosivo Intermitente (Look, McCloskey, y Coccaro, 2015).

La impulsividad y la búsqueda de sensaciones han sido frecuentemente vinculadas a comportamientos de riesgo en adolescentes, por ejemplo el consumo de sustancias (Gázquez et al., 2015). De hecho, una de las variables determinantes para el inicio en las drogas es la 
conducta impulsiva (Martínez-Loredo et al., 2016; Pérez-Fuentes et al., 2015), también relacionada con la conducta violenta en el ámbito escolar.(Pérez-Fuentes y Gázquez, 2010).

La capacidad para controlar los impulsos y la tendencia a la búsqueda de sensaciones, son a menudo analizadas de forma conjunta. En una muestra de adolescentes (Gjedde et al., 2010), empleando técnicas de neuroimagen, observaron que las puntuaciones en búsqueda de sensaciones, mantienen una relación invertida con respecto al número de receptores dopaminérgicos, asociados al sistema de recompensa cerebral, siendo esto último, la dificultad para demorar una recompensa, un rasgo característico de la respuesta impulsiva.

Peach y Gaultney (2013), proponen la tendencia a la búsqueda de sensaciones y la falla en el control de impulsos, como predictores del comportamiento violento en adolescentes. Estos autores, pudieron observar que la contribución relativa de la búsqueda de sensaciones disminuye desde la adolescencia hasta la edad adulta, mientras que la influencia del control de los impulsos es mayor con el paso del tiempo. Por su parte, Leeman, Hoff, Krishnan-Sarin, Patock-Peckham, y Potenza (2014), encontraron que, tanto la impulsividad como la búsqueda de sensaciones, estaban significativamente relacionadas con el consumo de sustancias y los juegos de azar en estudiantes de secundaria. Para Malmberg et al. (2013), la búsqueda de sensaciones y la impulsividad serían los dos rasgos que más peso tienen en el consumo de alcohol y tabaco, ya desde la adolescencia temprana. La primera, con mayor peso en el inicio del consumo, y la segunda, en su mantenimiento. Estos resultados apoyan la importancia de la búsqueda de sensaciones e impulsividad, para la adopción de comportamientos de riesgo entre los adolescentes (Alcázar, Verdejo, Bouso, y Ortega, 2015).

El objetivo del presente trabajo es conocer el valor predictivo de variables relacionadas con la búsqueda de sensaciones y con la impulsividad, en relación a la adopción de diferentes formas de agresión (agresión física, agresión verbal, ira y hostilidad), en adolescentes.

\section{Método}

\section{Participantes}

Teniendo en cuenta las diferentes zonas geográficas de la ciudad de Almería, se obtiene la muestra mediante muestro aleatorio por conglomerados, para lo que se seleccionan de forma aleatoria ocho centros de educación secundaria. El total de la muestra fue de 822 participantes, alumnos de $3^{\circ}$ y $4^{\circ}$ curso de Educación Secundaria Obligatoria (ESO), con un rango de edad de 13 a 18 años, y una media de 14.84 años $(D T=.87)$. Del total de la muestra, el $51.8 \%(N=426)$ eran hombres y el $48.2 \%(N=396)$ mujeres, con una media de edad de 14.85 años $(D T=.87)$ y 14.82 años $(D T=.86)$, respectivamente. La distribución de la muestra por curso académico fue la siguiente: el $43.7 \%$ eran alumnos de $3^{\circ} \mathrm{ESO}(N=359)$ y el $56.3 \%$ restante, se encontraba cursando $4^{\circ}$ ESO $(N=463)$.

\section{Instrumentos}

Escala de Búsqueda de Sensaciones (Pérez y Torrubia, 1986). Permite a partir de 40 ítems, con respuesta dicotómica (sí/ no), evaluar el rasgo de búsqueda de experiencias novedosas y arriesgadas. Se compone de cuatro subescalas: Búsqueda de Emociones (BEM), Búsqueda de 
Excitación (BEX), Desinhibición (DES) y Susceptibilidad hacia el Aburrimiento (SAB). Los autores obtienen coeficientes de fiabilidad que se sitúan entre .70 y .87 para las subescalas, y un alfa de Cronbach en torno al .87 para la escala total del cuestionario. En nuestro caso, se obtiene un coeficiente alfa de .76 para la escala completa, y valores que oscilan entre .40 y .72, para las subescalas.

Escala de Impulsividad Estado (EIE) de Iribarren, Jiménez-Giménez, García-de Cecilia y Rubio-Valladolid (2011). Diseñada para evaluar la conducta impulsiva definida como un estado, es decir, la impulsividad como una conducta manifiesta que puede variar a corto plazo. Consta de un total de 20 ítems de escala tipo likert, distribuidos en tres subescalas: Gratificación, Automatismo, y Atencional. Los autores (Iribarren et al., 2011) confirman una fiabilidad elevada, tanto para la escala completa $(\alpha=.88)$, como para cada una de sus dimensiones: Gratificación $(\alpha=.84)$, Automatismo $(\alpha=.80)$, y Atencional $(\alpha=.75)$. Para la muestra de estudio, se obtienen valores de alfa de .73 (Gratificación), .76 (Automatismo), y .80 (Atencional); coincidiendo con Iribarren et al (2011), con un $\alpha=.88$ para la escala total.

Aggresion Questionnaire (AQ) de Buss y Perry (1992).En este caso, se aplicó la adaptación española de Andreu, Peña, y Graña (2002). El cuestionario consta de 29 ítems y trata de evaluar la agresividad mediante cuatro factores: agresividad física, agresividad verbal, hostilidad e ira. Los 29 ítems están codificados en una escala tipo Likert de cinco puntos, donde: (1= completamente falso para mí; 2= bastante falso para mí; 3= ni verdadero ni falso para mí; 4= bastante verdadero para mí; 5= completamente verdadero para mí). Los coeficientes de fiabilidad encontrados en el estudio original de Buss y Perry, oscilaron entre .72 y .85. Para la adaptación española, los autores presentan un índice de fiabilidad para la escala completa de .88, mientras que para las escalas que lo integran el alfa de Cronbach osciló entre .68 y .86. En el presente trabajo, se obtiene un alfa de Cronbach de 0.87 para el cuestionario completo. Por otro lado, los valores obtenidos para cada una de las escalas, en este caso, son: $\alpha=.79$ en la Agresión física; $\alpha=.69$ en la Agresión verbal; $\alpha=.69$ para la Ira; y $\alpha=.68$ para la Hostilidad.

\section{Procedimiento}

En primer lugar, se informó a los responsables de cada centro sobre los objetivos, procedimiento y uso de los datos de la investigación. Además, se solicitaron los permisos pertinentes, mediante hoja de consentimiento informado dirigida a los padres/tutores. Antes de la implementación de las pruebas, se proporcionó a los participantes las instrucciones para su cumplimentación, así como la garantía de confidencialidad en el tratamiento de los datos. Posteriormente, dos miembros del equipo de investigación se desplazaron a los centros de Educación Secundaria, para la implementación de las pruebas.

\section{Análisis de datos}

En primer lugar, para identificar las variables relacionadas con las distintas formas de agresión analizadas (Agresión física, Agresión verbal, Ira y Hostilidad), se calculó el coeficiente de correlación de Pearson, además de los estadísticos descriptivos correspondientes. Para conocer cómo las variables predictoras (Búsqueda de sensaciones: Gratificación, Automatismo, y Atencional; Impulsividad: Búsqueda de emociones, Búsqueda de excitación, 
Desinhibición, y Susceptibilidad hacia el aburrimiento) se relacionan con cada una de las variables criterio, se lleva a cabo un análisis de regresión lineal múltiple por pasos (stepwise), para cada uno de los casos.

\section{Resultados}

Factores de Búsqueda de sensaciones e Impulsividad asociados a la agresión

Los coeficientes de correlación obtenidos, evidencian la existencia de correlaciones positivas entre los factores de búsqueda de sensaciones e impulsividad, con respecto a las modalidades de agresión analizadas.

Tal y como se observa en la tabla 1, los adolescentes con altas puntuaciones en agresión física, presentan altos niveles de Gratificación $(r=.38 ; p<.01)$, Automatismo $(r=.35 ; p<.01)$, Atencional $(r=.33 ; p<.01)$, Búsqueda de emociones $(r=.15 ; p<.01)$, Desinhibición $(r=.44$; $p<.01)$, y Susceptibilidad hacia el aburrimiento $(r=.20 ; p<.01)$.

Por otro lado, altas puntuaciones en agresión verbal, correlacionan positivamente con todos los factores de Impulsividad (GRA: $r=.41 ; p<.01$; AUTO: $r=.38$; $p<.01$; ATEN: $r=.38$; $p<.01$ ), y los relacionados con la Búsqueda de sensaciones (BEM: $r=.11 ; p<.01$; BEX: $r=.12 ; p<.01$; DES: $r=.31 ; p<.01$; SAB: $r=.27 ; p<.01$ ).

Tabla 1. Estadísticos descriptivos de los factores de impulsividad y búsqueda de sensaciones, y coeficientes de

\begin{tabular}{lcccccc}
\multicolumn{7}{c}{ correlación con las modalidades de agresión $(N=822)$} \\
\hline \multicolumn{1}{c}{ Impulsividad } & Media & $D T$ & $\begin{array}{c}\text { Correlación con } \\
\text { Agresión Física }\end{array}$ & $\begin{array}{c}\text { Correlación con } \\
\text { Agresión Verbal }\end{array}$ & $\begin{array}{c}\text { Correlación con } \\
\text { Ira }\end{array}$ & $\begin{array}{c}\text { Correlación con } \\
\text { Hostilidad }\end{array}$ \\
\hline GRA & 13.19 & 4.08 & $.38^{* *}$ & $.41^{* *}$ & $.41^{* *}$ & $.26^{* *}$ \\
AUTO & 11.86 & 3.93 & $.35^{* *}$ & $.38^{* *}$ & $.48^{* *}$ & $.31^{* *}$ \\
ATEN & 14.14 & 4.35 & $.33^{* *}$ & $.38^{* *}$ & $.51^{* *}$ & $.37^{* *}$ \\
\hline \multicolumn{1}{c}{$\begin{array}{l}\text { Búsqueda de } \\
\text { sensaciones }\end{array}$} & Media & $D T$ & $\begin{array}{c}\text { Correlación con } \\
\text { Agresión Física }\end{array}$ & $\begin{array}{c}\text { Correlación con } \\
\text { Agresión Verbal }\end{array}$ & $\begin{array}{c}\text { Correlación con } \\
\text { Ira }\end{array}$ & $\begin{array}{c}\text { Correlación con } \\
\text { Hostilidad }\end{array}$ \\
\hline BEM & 6.10 & 2.57 & $.15^{* *}$ & $.11^{* *}$ & $.06^{*}$ & .09 \\
BEX & 5.24 & 1.72 & .05 & $.12^{* *}$ & $.16^{* *}$ & $.17^{* *}$ \\
DES & 4.34 & 2.30 & $.44^{* *}$ & $.31^{* *}$ & $.26^{* *}$ & $.19^{* *}$ \\
SAB & 4.22 & 1.95 & $.20^{* *}$ & $.27^{* *}$ & $.27^{* *}$ & $.18^{* *}$
\end{tabular}

"Correlación significativa al nivel .05; ${ }^{* *}$ Correlación significativa al nivel .01.

Nota: GRA= Gratificación; AUTO= Automatismo; ATEN= Atencional; BEM= Búsqueda de emociones; BEX= Búsqueda de excitación; DES= Desinhibición; $\mathrm{SAB}=$ Susceptibilidad hacia el aburrimiento.

En lo que se refiere a la Ira, como componente de la agresión, en la tabla 1 se puede observar cómo se dan correlaciones con todos los componentes de Impulsividad (GRA: $r=.41$; $p<.01$; AUTO: $r=.48$; $p<.01$; ATEN: $r=.51 ; p<.01$ ), y de la Búsqueda de sensaciones (BEM: $r=.06$; $p<.05$; BEX: $r=.16$; $p<.01$; DES: $r=.26$; $p<.01$; SAB: $r=.27 ; p<.01$ ).

Finalmente, vistos los coeficientes de correlación obtenidos para la hostilidad, se observa que los adolescentes con altas puntuaciones en esta forma de agresión, presentan también altas 
puntuaciones en Gratificación ( $r=.26 ; p<.01)$, Automatismo $(r=.31 ; p<.01)$, Atencional $(r=.37$; $p<.01)$, Búsqueda de excitación $(r=.17 ; p<.01)$, Desinhibición $(r=.19 ; p<.01)$, y Susceptibilidad hacia el aburrimiento $(r=.18 ; p<.01)$.

\section{Variables predictoras de la Agresión Física}

Según los datos recogidos en la tabla 2, el análisis de regresión arroja tres modelos, donde el modelo 3 es el que mayor capacidad explicativa ofrece, con un $26 \%\left(R^{2}=.26\right)$ de la varianza explicada por los factores incluidos en el modelo.

Tabla 2. Modelo de Regresión Lineal Múltiple por pasos(Agresión física)

\begin{tabular}{|c|c|c|c|c|c|c|c|c|}
\hline \multirow[b]{2}{*}{ Modelo } & \multirow[b]{2}{*}{$R$} & \multirow[b]{2}{*}{$R^{2}$} & \multirow{2}{*}{$\begin{array}{c}R^{2} \\
\text { corregida }\end{array}$} & \multicolumn{4}{|c|}{ Estadísticos de cambio } & \multirow{2}{*}{$\begin{array}{l}\text { Durbin } \\
\text { Watson }\end{array}$} \\
\hline & & & & $\begin{array}{l}\text { Error típico de } \\
\text { estimación }\end{array}$ & $\begin{array}{c}\text { Cambio en } \\
R^{2}\end{array}$ & $\begin{array}{c}\text { Cambio en } \\
F\end{array}$ & $\begin{array}{l}\text { Sig. del cambio en } \\
\qquad F\end{array}$ & \\
\hline 1 & .45 & .20 & .20 & .74 & .20 & 204.95 & .00 & \\
\hline 2 & .50 & .26 & .25 & .72 & .06 & 59.92 & .00 & 1.83 \\
\hline 3 & .51 & .26 & .26 & .72 & .01 & 6.45 & .01 & \\
\hline
\end{tabular}

Para confirmar la validez del modelo, se analizó la independencia de los residuos. El estadístico $D$ de Durbin-Watson obtiene un valor $D=1.83$, que confirma la ausencia de autocorrelación positiva y negativa.

En la tabla 3, se observa como el valor de $T$ se asocia a una probabilidad de error inferior al .05 en todas las variables incluidas en el modelo. Además, los coeficientes estandarizados revelan que las variables que presentan un mayor peso explicativo son: la Desinhibición, el Automatismo y la Gratificación. Las puntuaciones obtenidas, sitúan al factor Desinhibición como el predictor más fuerte de la agresión física.

\begin{tabular}{|c|c|c|c|c|c|c|c|}
\hline \multirow{2}{*}{ Modelo } & \multicolumn{2}{|c|}{ Coeficientes no estandarizados } & \multirow{2}{*}{$\begin{array}{c}\text { Coeficientes tipificados } \\
\text { Beta }\end{array}$} & \multirow{2}{*}{$T$} & \multirow{2}{*}{ Sig. } & \multicolumn{2}{|c|}{ Colinealidad } \\
\hline & $B$ & Error típ. & & & & Tol. & FIV \\
\hline (Constante) & 1.17 & .09 & & 12.80 & .00 & & \\
\hline DES & .12 & .01 & .34 & 9.89 & .00 & .77 & 1.30 \\
\hline AUTO & .04 & .01 & .19 & 5.27 & .00 & .66 & 1.52 \\
\hline GRA & .02 & .01 & .10 & 2.54 & .01 & .56 & 1.80 \\
\hline
\end{tabular}

Finalmente, se asume la ausencia de colinealidad entre las variables incluidas en el modelo, al obtener altos valores en los indicadores de tolerancia y bajos en FIV. 


\section{Variables predictoras de la Agresión Verbal}

En la tabla 4, se muestran los cinco modelos que resultan del análisis de regresión, donde el modelo 5 es el que obtiene un porcentaje de varianza explicada del $24 \%\left(\mathrm{R}^{2}=.24\right)$. En este caso, el estadístico $D$ de Dubin-Watson, confirma la validez del modelo $(D=1.95)$.

Tabla 4. Modelo de Regresión Lineal Múltiple por pasos (Agresión verbal)

\begin{tabular}{|c|c|c|c|c|c|c|c|c|}
\hline \multirow[b]{2}{*}{ Modelo } & \multirow[b]{2}{*}{$R$} & \multirow{2}{*}{$R^{2}$} & \multirow{2}{*}{$\begin{array}{c}R^{2} \\
\text { corregida }\end{array}$} & \multicolumn{4}{|c|}{ Estadísticos de cambio } & \multirow{2}{*}{$\begin{array}{l}\text { Durbin } \\
\text { Watsor }\end{array}$} \\
\hline & & & & $\begin{array}{l}\text { Error típico de } \\
\text { estimación }\end{array}$ & $\begin{array}{c}\text { Cambio en } \\
R^{2}\end{array}$ & $\begin{array}{c}\text { Cambio en } \\
F\end{array}$ & $\begin{array}{c}\text { Sig. del cambio en } \\
F\end{array}$ & \\
\hline 1 & .41 & .17 & .17 & .70 & .17 & 166.00 & .00 & \\
\hline 2 & .45 & .20 & .20 & .69 & .03 & 33.36 & .00 & \\
\hline 3 & .47 & .22 & .22 & .68 & .02 & 17.73 & .00 & 1.95 \\
\hline 4 & .48 & .23 & .23 & .68 & .01 & 11.13 & .01 & \\
\hline 5 & .49 & .24 & .24 & .68 & .01 & 10.23 & .01 & \\
\hline
\end{tabular}

En cuanto al valor del estadístico $T$, en la tabla 5, se detecta una asociación a una probabilidad de error inferior a .05, para todas las variables incluidas en el modelo: Gratificación, Atencional, Susceptibilidad hacia el aburrimiento, Desinhibición y Automatismo. Según los valores obtenidos en los coeficientes estandarizados, la variable Gratificación se presenta como el predictor más fuerte de la Agresión verbal, seguido por el factor Atencional.

Tabla 5. Coeficientes del Modelo de Regresión Lineal Múltiple (Agresión verbal)

\begin{tabular}{|c|c|c|c|c|c|c|c|}
\hline \multirow{2}{*}{ Modelo } & \multicolumn{2}{|c|}{ Coeficientes no estandarizado } & \multirow{2}{*}{$\begin{array}{c}\text { Coeficientes tipificados } \\
\text { Beta }\end{array}$} & \multirow{2}{*}{$T$} & \multirow{2}{*}{ Sig. } & \multicolumn{2}{|c|}{ Colinealidad } \\
\hline & $B$ & Error típ. & & & & Tol. & FIV \\
\hline (Constante) & 1.27 & .09 & & 13.68 & .00 & & \\
\hline GRA & .03 & .01 & .16 & 3.79 & .00 & .51 & 1.96 \\
\hline ATEN & .02 & .01 & .13 & 2.97 & .01 & .50 & 1.99 \\
\hline $\mathrm{SAB}$ & .04 & .01 & .11 & 3.36 & .01 & .86 & 1.17 \\
\hline DES & .04 & .01 & .12 & 3.45 & .01 & .74 & 1.36 \\
\hline AUTO & .03 & .01 & .14 & 3.20 & .01 & .50 & 1.99 \\
\hline
\end{tabular}

Nota: GRA= Gratificación; ATEN= Atencional; SAB= Susceptibilidad hacia el aburrimiento; DES= Desinhibición; AUTO= Automatismo.

En vista de los valores obtenidos para los indicadores de Tolerancia y FIV, en este caso, se asume la ausencia de colinealidad entre las variables Susceptibilidad hacia el aburrimiento y Desinhibición, al obtener altos valores en los indicadores de tolerancia y bajos en FIV. El resto de las variables incluidas en el modelo, a pesar de no rebasar los límites estipulados para estos indicadores, quedan muy próximos a la presencia de cierta colinealidad. 


\section{Variables predictoras de la Ira}

Tal y como se presenta en la tabla 6, para la Ira como variable de entrada, se obtienen cinco modelos. En este caso, el modelo 5 es el que mayor capacidad explicativa ofrece. Por tanto, el $32 \%$ de la varianza $\left(R^{2}=.32\right)$ en Ira, es explicada por las variables incluidas en el modelo: Atencional, Automatismo, Susceptibilidad hacia el aburrimiento, Búsqueda de excitación y Gratificación. Asimismo, la validez del modelo queda reflejada en la independencia de los residuos, con un valor $D=1.89$ para el estadístico de Durbin-Watson.

Tabla 6. Modelo de Regresión Lineal Múltiple por pasos (Ira)

\begin{tabular}{|c|c|c|c|c|c|c|c|c|}
\hline \multirow[b]{2}{*}{ Modelo } & \multirow[b]{2}{*}{$R$} & \multirow{2}{*}{$R^{2}$} & \multirow{2}{*}{$\begin{array}{c}R^{2} \\
\text { corregida }\end{array}$} & \multicolumn{4}{|c|}{ Estadísticos de cambio } & \multirow{2}{*}{$\begin{array}{l}\text { Durbin } \\
\text { Watsor }\end{array}$} \\
\hline & & & & $\begin{array}{l}\text { Error típico de } \\
\text { estimación }\end{array}$ & $\begin{array}{c}\text { Cambio en } \\
R^{2}\end{array}$ & $\begin{array}{c}\text { Cambio en } \\
F\end{array}$ & $\begin{array}{c}\text { Sig. del cambio en } \\
F\end{array}$ & \\
\hline 1 & .52 & .27 & .27 & .71 & .27 & 300.81 & .00 & \\
\hline 2 & .55 & .30 & .30 & .69 & .04 & 41.31 & .00 & \\
\hline 3 & .56 & .32 & .32 & .69 & .01 & 16.97 & .00 & 1.89 \\
\hline 4 & .57 & .32 & .32 & .69 & .01 & 5.77 & .02 & \\
\hline 5 & .57 & .33 & .32 & .69 & .01 & 4.09 & .04 & \\
\hline
\end{tabular}

Por su parte, el estadístico $T$ se asocia a una probabilidad de error inferior a .05 en las cinco variables incluidas en el modelo (Tabla 7). Y, atendiendo a los coeficientes estandarizados, las variables que presentan un mayor peso explicativo son: Atención, Automatismo, Susceptibilidad hacia el aburrimiento, Búsqueda de excitación y Gratificación. Estos coeficientes, sitúan a la variable Atencional como el predictor más fuerte de la Ira, seguido por el Automatismo.

Tabla 7. Coeficientes del Modelo de Regresión Lineal Múltiple (Ira)

\begin{tabular}{|c|c|c|c|c|c|c|c|}
\hline \multirow{2}{*}{ Modelo } & \multicolumn{2}{|c|}{ Coeficientes no estandarizados } & \multirow{2}{*}{$\begin{array}{c}\text { Coeficientes tipificados } \\
\text { Beta }\end{array}$} & \multirow{2}{*}{$T$} & \multirow{2}{*}{ Sig. } & \multicolumn{2}{|c|}{ Colinealidad } \\
\hline & $B$ & Error típ. & & & & Tol. & FIV \\
\hline (Constante) & 1.03 & .11 & & 9.33 & .00 & & \\
\hline ATEN & .06 & .01 & .30 & 7.33 & .00 & .50 & 2.00 \\
\hline AUTO & .04 & .01 & .21 & 5.05 & .00 & .50 & 1.99 \\
\hline SAB & .05 & .01 & .11 & 3.52 & .00 & .88 & 1.14 \\
\hline BEX & .03 & .01 & .07 & 2.27 & .02 & .96 & 1.04 \\
\hline GRA & .02 & .01 & .08 & 2.02 & .04 & .57 & 1.74 \\
\hline
\end{tabular}

Nota: ATEN= Atencional; $\mathrm{AUTO}=$ Automatismo; $\mathrm{SAB}=$ Susceptibilidad hacia el aburrimiento; $\mathrm{BEX}=\mathrm{Búsqueda}$ de excitación; GRA= Gratificación. 
Por último, en este caso, se asume la ausencia de colinealidad entre las variables Susceptibilidad hacia el aburrimiento, Búsqueda de excitación y Gratificación, al obtener altos valores en los indicadores de tolerancia y bajos en FIV. El resto de las variables incluidas en el modelo, a pesar de no rebasar los límites previstos para estos indicadores, quedan muy próximos a la presencia de cierta colinealidad.

\section{Variables predictoras de la Hostilidad}

Tal y como se muestra en la tabla 8 , como resultado del análisis de regresión múltiple, se obtienen tres modelos, donde el modelo 3 es el que mayor capacidad explicativa ofrece, con un $16 \%\left(R^{2}=.16\right)$ de la varianza explicada por los factores incluidos en el modelo.

Para confirmar la validez del modelo, se analizó la independencia de los residuos. Se obtiene un valor $D=1.92$ para el estadístico $D$ de Durbin-Watson, que confirma la ausencia de autocorrelación positiva y negativa.

Tabla 8. Modelo de Regresión Lineal Múltiple por pasos (Hostilidad)

\begin{tabular}{|c|c|c|c|c|c|c|c|c|}
\hline \multirow[b]{2}{*}{ Modelo } & \multirow[b]{2}{*}{$R$} & \multirow[b]{2}{*}{$R^{2}$} & \multirow{2}{*}{$\begin{array}{c}R^{2} \\
\text { corregida }\end{array}$} & \multicolumn{4}{|c|}{ Estadísticos de cambio } & \multirow{2}{*}{$\begin{array}{l}\text { Durbin } \\
\text { Watsor }\end{array}$} \\
\hline & & & & $\begin{array}{l}\text { Error típico de } \\
\text { estimación }\end{array}$ & $\begin{array}{c}\text { Cambio en } \\
R^{2}\end{array}$ & $\begin{array}{c}\text { Cambio en } \\
F\end{array}$ & $\begin{array}{c}\text { Sig. del cambio en } \\
F\end{array}$ & \\
\hline 1 & .38 & .14 & .14 & .69 & .14 & 135.37 & .00 & \\
\hline 2 & .39 & .15 & .15 & 69 & .01 & 11.76 & .01 & 1.92 \\
\hline 3 & .40 & .16 & .16 & .68 & .01 & 8.46 & .01 & \\
\hline
\end{tabular}

En la tabla 9, se observa como el valor de $T$ se asocia a una probabilidad de error inferior al .05 en todas las variables incluidas en el modelo. Además, los coeficientes estandarizados revelan que el factor Atencional es el predictor más fuerte de la hostilidad.

Tabla 9. Coeficientes del Modelo de Regresión Lineal Múltiple (Hostilidad)

\begin{tabular}{|c|c|c|c|c|c|c|c|}
\hline \multirow{2}{*}{ Modelo } & \multicolumn{2}{|c|}{ Coeficientes no estandarizados } & \multirow{2}{*}{$\begin{array}{c}\text { Coeficientes tipificados } \\
\text { Beta }\end{array}$} & \multirow{2}{*}{$T$} & \multirow{2}{*}{ Sig. } & \multicolumn{2}{|c|}{ Colinealidad } \\
\hline & $B$ & Error típ. & & & & Tol. & FIV \\
\hline (Constante) & 1.74 & .10 & & 16.66 & .00 & & \\
\hline ATEN & .05 & .01 & .28 & 6.43 & .00 & .56 & 1.80 \\
\hline SAB & 0.48 & .01 & .11 & 3.44 & .01 & .97 & 1.03 \\
\hline AUTO & 0.23 & .01 & .12 & 2.91 & .01 & .57 & 1.77 \\
\hline
\end{tabular}

Nota: ATEN= Atencional; $\mathrm{SAB}=$ Susceptibilidad hacia el aburrimiento; AUTO= Automatismo.

Finalmente, se asume la ausencia de colinealidad entre las variables incluidas en el modelo, al obtener altos valores en los indicadores de tolerancia y bajos en FIV. 


\section{Discusión y conclusiones}

Los resultados obtenidos evidencian la existencia de una relación positiva entre los factores relacionados con la búsqueda de sensaciones y la impulsividad (Archer y Webb, 2006), con respecto a las diferentes formas de agresión, contempladas en el cuestionario de Buss y Perry. Así, los adolescentes que incluyen la agresión física y verbal, la ira y la hostilidad (Inglés et al, 2014; McKay et al., 2016), como respuesta habitual en su repertorio conductual, coinciden en una serie de características: a) relacionadas con la impulsividad (urgencia en la satisfacción de impulsos, tienden a actuar sin tener en cuenta las consecuencias negativas o la información que les proporcionan las variables contextuales, ausencia de planificación), y b) relacionadas con la búsqueda de sensaciones (necesidad de tener nuevas experiencias y sensaciones variadas, y tendencia a arriesgarse para conseguirlo).

Además, con el presente trabajo, se ha tratado de conocer el valor predictivo de variables relacionadas con la búsqueda de sensaciones y la impulsividad, en relación a diferentes componentes de la agresión, en adolescentes. Los resultados obtenidos a partir del análisis de regresión múltiple, revelan que en todos los casos, la conducta de agresión queda explicada mediante la combinación de diferentes factores de la búsqueda de sensaciones y de la impulsividad (Peach y Gaultney, 2013). Esto apoya las propuestas de diferentes estudios (Gjedde et al., 2010; Platje, 2013), donde se aboga por el estudio conjunto de los efectos de la capacidad para controlar los impulsos y la tendencia a experimentar nuevas sensaciones, sobre el comportamiento violento en adolescentes (Peach y Gaultney, 2013).

Concretamente, en nuestro caso, la Desinhibición sería el factor que mejor explica la agresión física, en combinación con dos rasgos de la impulsividad, como el Automatismo y la Gratificación (Muñoz et al., 2005).En la agresión verbal, destaca la Gratificación como el factor de mayor valor explicativo, junto al resto de componentes de la impulsividad (Neumann et al., 2010), y además la Desinhibición y Susceptibilidad hacia el aburrimiento. La presencia de los componentes de la impulsividad en los modelos explicativos de la agresión física y verbal, coinciden con lo que otros autores (Coccaro et al., 2014; Look et al., 2015) han observado acerca de la presencia de altos niveles de impulsividad en sujetos con este tipo de conductas.

Por otro lado, destaca el factor Atencional como el mejor predictor, presente en los modelos explicativos de la ira y la hostilidad. Además, en ambos casos, otros factores de la impulsividad (Chen et al., 2012) y de la búsqueda de sensaciones (Moral y Ortiz, 2011), están presentes.

El hecho de que algunos de los factores, tanto de la impulsividad como de búsqueda de sensaciones, muestren un valor explicativo en varias de las formas de agresión analizadas, pone en relieve la interrelación que se da entre éstas. Resultados que van en consonancia con los obtenidos en investigaciones recientes (Rubio-Garay et al., 2016), donde la ira y la hostilidad mantienen, a su vez, un cierto grado de influencia sobre las otras dos manifestaciones de la agresión: la física y la verbal.

Como conclusión, destacar la importancia de continuar la labor de investigación en cuanto a la obtención de datos acerca de las variables explicativas de conductas de riesgo en adolescentes. Por tratarse ésta de una etapa repleta de cambios, a todos los niveles, será necesario atender especialmente a las características psicológicas del adolescente y a los 
efectos que puedan tener sobre su conducta (Galvan, 2010). Este es el caso del control de impulsos y la tendencia hacia la búsqueda de sensaciones, que se presentan como predictores de la asunción de conductas de riesgo en esta etapa (Pérez-Fuentes et al., 2015), entre ellas las diferentes formas de expresión que adopta la conducta violenta.

Agradecimientos: El presente estudio cuenta con la colaboración de la Excma. Diputación Provincial de Almería.

\section{Referencias}

Alcázar, M.A., Verdejo, A., Bouso, J.C., y Ortega, J. (2015). Búsqueda de sensaciones y conducta antisocial. Anuario de Psicología Jurídica, 25(1), 75-80.

Álvarez, A., Suárez, N., Tuero, E., Núñez, J., Valle, A., y Regueiro, B. (2015). Implicación familiar, autoconcepto del adolescente y rendimiento académico. European Journal of Investigation in Health, Psychology and Education, 5(3), 293-311.

Andreu, J.M., Peña, M.E., \& Graña, J.L. (2002). Psychometric adaptation of the Spanish version of the Aggression Questionnaire. Psicothema, 14(2), 476-482.

Archer, J. y Webb, I.A. (2006). The relation between scores on the Buss-Perry Aggression Questionnaire and aggressive acts, impulsiveness, competitiveness, dominance, and sexual jealousy. Aggressive Behavior, 32(5), 464-473.

Azpiazua, L., Esnaola, I., y Sarasa, M. (2015). Capacidad predictiva del apoyo social en la inteligencia emocional de adolescentes. European Journal of Education and Psychology, $8(1), 23-29$.

Bandura, A. (1976). Social learning analysis of aggression. En E. Ribes y A. Bandura (Eds.), Analysis of delinquency and aggression (pp. 203-232). Hillsdale: Erlbaum.

Buss, A.H. (1961). The psychology of aggression. New York: Wiley.

Buss, A.H., y Perry, M.P. (1992). The aggression questionnaire. Journal of Personality and Social Psychology, 63(3), 452-459.

Calvet, M.M., Yaringaño, J., Uceda, J., Fernández, E., Huari, Y., y Campos, A. (2012). Estudio psicométrico de la versión española del cuestionario de agresión de Buss y Perry. Revista de Investigación en Psicología, 15(1), 147-161.

Cardoso-Moreno, M.J., Tomás-Aragonés, L., y Rodríguez-Ledo, C. (2015). Socio-emotional intervention in attention deficit hyperactive disorder. European Journal of Education and Psychology, 8(2), 53-59.

Chen, P., Coccaro, E.F., y Jacobson, K.C. (2012). Hostile attributional bias, negative emotional responding, and aggression in adults: Moderating effects of gender and impulsivity. Aggressive Behavior, 38(1), 47-63.

Coccaro, E.F., Lee, R., y McCloskey, M.S. (2014). Relationship between psychopathy, aggression, anger, impulsivity, and intermittent explosive disorder. Aggressive Behavior, 40(6), 526-536.

Galvan, A. (2010). Adolescent development of the reward system. Frontiers Human Neuroscience, 4(6), 1-9. 
Gázquez, J., Pérez-Fuentes, M., Molero, M., Martos, Á., Cardila, F., Barragán, A., Carrión, J., Garzón, A., y Mercader, I. (2015). Adaptación Española del Cuestionario de Expectativas del Alcohol en Adolescentes. European Journal of Investigation in Health, Psychology and Education, 5(3), 357-369.

Gjedde, A., Kumakura, Y., Cumming, P., Linnet, J., y Moller, A. (2010). Inverted-U-shaped correlation between dopamine receptor availability in striatum and sensation seeking. PNAS, 107(8), 3870-3875.

Inglés, C.J., Gonzálvez-Maciá, C., García-Fernández, J.M., Vicent, M., y MartínezMonteagudo, M.C. (2015). Current status of research on school refusal. European Journal of Education and Psychology, 8(1), 37-52.

Inglés, C.J., Torregrosa, M.S., García-Fernández, J.M., Martínez-Monteagudo, M.C., Estévez, E., y Delgado, B. (2014). Conducta agresiva e inteligencia emocional en la adolescencia. European Journal of Education and Psychology, 7(1), 29-41.

Iribarren, M.M., Jiménez-Giménez, M., García-de Cecilia, J.M., y Rubio-Valladolid, G. (2011). Validación y propiedades psicométricas de la Escala de Impulsividad Estado (EIE). Actas Españolas de Psiquiatría, 39(1), 49-60.

Leeman, R.F., Hoff, R.A., Krishnan-Sarin, S., Patock-Peckhan, J.A., y Potenza, M.N. (2014). Impulsivity, Sensation-Seeking, and Part-Time Job Status in Relation to Substance Use and Gambling in Adolescents. Journal of Adolescent Health, 54(4), 460-466.

Look, A.E., McCloskey, M.S., y Coccaro, E.F. (2015). Verbal versus physical aggression in Intermittent Explosive Disorder. Psychiatry Research, 225(3), 531-9.

Martínez-Loredo, V., Fernández-Artamendi, S., Weidberg, S., Pericot, I., López-Núñez, C., Fernández-Hermida, J., y Secades, R. (2016). Parenting styles and alcohol use among adolescents: A longitudinal study. European Journal of Investigation in Health, Psychology and Education, 6(1), 27-36.

McKay, M.T., Perry, J.L., y Harvey, S.A. (2016). The factorial validity and reliability of three versions of the Aggression Questionnaire using Confirmatory Factor Analysis and Exploratory Structural Equation Modelling. Personality and Individual Differences, 90, 12-15.

Moral de la R., J. y Ortiz M., H. (2011). Modelos predictivos de conducta disocial por sexos en dos colonias populares. Salud Mental, 34, 227-235.

Muñoz G., J.J., Navas C., E. y Graña G., J.L. (2005). Factores psicológicos de riesgo y protección para la conducta antisocial en adolescentes. Actas Españolas de Psiquiatría, 33(6), 366-373.

Neumann, A., Barker, E., Koot, H.M., y Maughan, B. (2010). The role of contextual risk, impulsivity, and parental knowledge in the development of adolescent antisocial behavior. Journal of Abnormal Psychology, 119(3), 534-545.

Peach, H.D. y Gaultney, J.F. (2013). Sleep, Impulse Control, and Sensation-Seeking Predict Delinquent Behavior in Adolescents, Emerging Adults, and Adults. Journal of Adolescent Health, 53(2), 293-299.

Pérez, J. y Torrubia, R. (1986). Fiabilidad y validez de la versión española de la Escala de Búsqueda de Sensaciones (Forma V). Revista Latinoamericana de Psicología, 18(1), 722. 
Pérez-Fuentes, M., Gázquez, J., Molero, M., Cardila, F., Martos, A., Barragán, A., Garzón, A., Carrión, J., y Mercader, I. (2015). Impulsividad y consumo de alcohol y tabaco en adolescentes. European Journal of Investigation in Health, Psychology and Education, 5(3), 371-382.

Platje, E., Vermeiren, R.R.J.M., Raine, A., Doreleijers, T.A.H., Keijsers, L.G.M.T., Branje, S.J.T., y Jansen, L.M.C. (2013). A longitudinal biosocial study of cortisol and peer influence on the development of adolescent antisocial behaviour. Psychoneuroendocrinology, 38(11), 2770-2779.

Rubio-Garay, F., Carrasco, M.A., y Amor, P.J. (2016). Aggression, anger and hostility: evaluation of moral disengagement as a meditational process. Scandinavian Journal of Psychology [versión online].

Zuckerman, M., Persky, H, Link, K. y Basu, G.K. (1994). Experimental and subject factors determining responses to sensory deprivation, social isolation, and confinement. Journal of Abnormal Psychology, 73(3), 183. 